\title{
On the Connection Between the Properties of Oriented Linear Graphs and Analyses of Lumped Physical Systems
}

\author{
Horace M. Trent ${ }^{1}$ \\ U.S. Naval Research Laboratory
}

(February 17, 1965)

It is necessary at the outset of this lecture to apologize to this assemblage of mature theoreticians for talking about an essentially engineering subject. My excuse for doing so is that whereas my subject represents one of the most important current applications of the theory of linear graphs, it is all too clear from the literature that a number of misconceptions about it are being passed along from person to person. A word of warning is in order also. The subject I am to discuss is like a multifaced gem in that it has many facets, each of which can add to an appreciation of the beauty of the whole object. Indeed whole books have been written on a restricted aspect of the total subject; the application of linear graphs to electric networks for example. This being the case, it is foolish to think that I can give a definitive exposition of the subject in less than an hour. At most, it will be possible only to touch upon those ideas and concepts which either are rather basic or are often overlooked by some people who use the techniques.

It is well at the outset to bear in mind that the theory of linear graphs is used, in the application under discussion, as an aid, and as a unifying concept in the analysis of what can be called hereafter, an engineering system. In particular, the techniques are applicable to those engineering systems which can be described, with adequate precision, by a finite number of physical variables. This limitation assures us that we shall be dealing only with finite linear graphs as will become more evident later. These remarks suggest, or rather demand, that we look carefully at engineering analyses and extract from them those concepts and operations that are pertinent to the problem at hand. By this it is meant that our problem is to justify, in some logical fashion, just how the properties of linear graphs, which after all are only lines on a sheet of paper, can be used in a meaningful way in the analysis of a finite engineering system. Surely no one in this audience believes that a linear graph drawn on the blackboard in this room and, say, a motor-generator down the hall are the same object. And surely more than one person here is wondering why such a trite remark has been made. There are two reasons. In the first place some trite remarks emphasize fundamental concepts.

${ }^{1}$ Dr. Trent died December 16, 1964. He was Head of Applied Mathematics at NRL.
Such a case is at hand for it is absolutely basic to understand that a linear graph and an engineering system under analysis are distinct objects. Second, the remark is made to counteract certain misconceptions that appear in the open literature. For example, one well known book contains a sentence which begins, "we define an electric network to be a linear graph, etc." For goodness sake, an electric network cannot be defined to be anything but an electric network. In fact, it couldn't care less how anyone defines it. That is another trite statement full of deep meaning for an electric network, or any engineering system for that matter, will go on performing its own function in its own peculiar way no matter what a theoretician down the hall has to say about it. Hence a theoretician, if he is a good one, will not try to warp the characteristics of a system to fit his own whims and notions; rather he can only hope that his whims and notions may have some reasonable correspondence to what goes on in the real system.

Using the example of the motor-generator set down the hall, it is obvious that anything a theoretician does at his desk has no reaction whatsoever with the system under analysis. In other words, the performance of the motor-generator is completely unaffected by anything an analyst does with a pencil and a pad of paper. These remarks point up the fact that an analysis of an engineering system is strictly a mental exercise indulged in by a theoretician. Failure to recognize this fact can lead to unrealistic concepts; it has done so not too infrequently in the past.

It should be apparent at this point that if a linear graph is to be useful at all in the analysis of an engineering system it is highly likely that it can do so only by virtue of an isomorphism between some, but not all, of its properties and some of the mental objects which are created in the course of analyzing a system. The remainder of the remarks to be made today show in outline how such an isomorphism can be established.

Let us begin by looking at the pertinent mental exercises indulged in by an analyst. The order in which these mental operations will be discussed is not necessarily the order in which they are done in a specific application.

First, some assumptions must be made about the nature of space and time. It is often assumed for 
example that space is Euclidean and that time is isochronous. It would be good if it could be asserted that analysts are aware of their making such assumptions each time they do so; but unfortunately this is not the case. Nevertheless, these assumptions are inherent in most engineering analyses.

Next the analyst looks at those energy mechanisms which exist in his system under analysis. He then must place each mechanism into one of three categories; (1) the mechanism is judged to be unimportant and hence is excluded from the analysis, (2) the mechanism is too significient to be ignored, yet there is no need to analyze it in detail, in which case it is covered up by some artful dodge, and (3) the mechanism is significant and it is desirable to analyze it in detail. Again using the motor-generator set as an example, the magnetostrictive effect, which always exists to some degree in the laminations of the magnet, probably is insignificant and can be excluded from the analysis of the system without introducing an appreciable error. The details of what happens to the heat generated by the current flowing through the windings on the rotor may not be of significance. The total amount of energy involved in this mechanism probably is too large to be ignored so it must be included in the analysis, but not in detail, by assigning a resistance to the winding. A similar coverup job is usually done with respect to the magnetic fields established in the set by a magnet of some sort. Finally it is usually the case that there is great interest in the electrical energy supplied to and extracted from the system and in its mechanical behavior. These two energy mechanisms are then analyzed in detail; but note that this is so only because of a primary interest in the details of these mechanisms.

Having identified those mechanisms which are to be analyzed in detail, the analyst is then faced with the problem of selecting variables which are appropriate for describing these mechanisms. It is to be emphasized at this point that the information that the analyst desires is contained in these variables; hence their selection is not a matter to be treated lightly.

It is observed first that for any one mechanism two kinds of variables are required. Again citing the motor-generator set, the electrical portion of the system is usually described in terms of voltage drops and electric currents although such a selection is not mandatory. A voltage drop is typical of a class of variable which Firestone called across variables. Mathematicians usually call them contravariant variables. Their important characteristic is that they are defined in terms of two terminals in the system where by a terminal is meant a point or a surface needed to define the variable. Conceptually any across variable can be measured by an appropriate instrument attached to two terminals. Currents, on the other hand, are typical of a second type of variable; one which acts as if something is propagated through the system. The phrase "as if" is intended to imply, to paraphrase a comment of Faraday, "the variable may not represent the actual propagation of something through the system; but if it did, the system would act just like it does." Firestone called these through variables while mathematicians call them covariant variables. Any one variable of this type can be measured, at least conceptually, by breaking the system at a terminal and then inserting an appropriate meter.

Having made a selection of variables for one sort of energy mechanism, an analyst no longer has complete freedom in selecting variables for another mechanism. Note that in the illustration cited above, the product of the two variables selected to describe the electrical portion of the system, namely voltage drops and currents, has the physical dimensions of power. This fact leads to the requirement that appropriate variables for the purely mechanical portions of the system must yield the same sort of a product. Thus torques and angular velocities might be used as appropriate variables.

Having selected appropriate variables, the analyst then conceptually decomposes the system into a set of simple elements. By a simple element is meant a conceptual object, with identified terminals, whose performance is describable by a known set of relations involving the variables already selected. These relations are called Constitutive Equations.

Quite a lot of things can be said about elements but only a few items can be discussed here. First of all an analyst locates the terminals of the device. In this process certain points or surfaces, which seem not to be a part of the element, are needed to define some of the across variables. A very simple example arises with the rigid mass in rectilinear motion with its constitutive equation $f=m \frac{d v}{d t}$. This equation is true only

if the across variable, $v$, is defined between some point on, or in, the rigid body, the center of mass for example, and an inertial reference. The inertial reference at first glance seems not to be a part of the element but this point of view is not correct. Situations analogous to the foregoing are common. Terminals which seem not to be in the element are called External References.

Next an analyst will seek to find a set of independent across variables for the element. He will try to select them so as to be the most advantageous in terms of those bits of information which he is seeking. Without going into the details, bounds can be set on the number of such variables in terms of the number of terminals on the element. If $V$ is the number of independent across variables associated with the element and $T$ is the number of terminals, then $T / 2 \leqslant V \leqslant$ $(T-1)$.

Having established a set of across variables defined as a set of terminals, a graphical symbol for the element is created by the following procedure. (1) A set of open or solid circles equal in number to the terminals on the element are put on a sheet of paper and each circle is identified with one specific terminal. (2) One line segment is drawn for each independent across variable, the line terminating on the pair of circles which correspond to the terminals of the device between which the variable is defined. (3) Certain portions of each line segment are replaced by standardized adorn- 
ments which serve to show the nature of the element which stands in correspondence to the graphical symbol. Koenig has called the process of adding the adornments, coding the symbol. This is an apt designation. It should be borne in mind that the coding of a graphical symbol implies the constitutive equation for the element. Thus if the coding is a sawtoothed line, it is generally known that the element is a resistor with a constitutive equation $e=i R$ or $i=G e$. The ability to write a constitutive equation in several ways is quite the rule rather than the exception.

We come now to two properties of through variables, neither of which can be proven from first principles. They represent the experience of the scientific world to date and in no case where engineering systems can be analyzed has an exception been found. First there are as many through variables associated with an element as there are across variables. Thus a single line in a graphical symbol stands in 1-to-1 correspondence with one across and one through variable. Second, the through variables act as if they are transmitted unchanged from one terminal to another along the same conceptual path suggested by the line segment. If the through variables leaving an element are considered positive and those entering negative, the foregoing continuity principle leads to the result that the algebraic sum of the through variables leaving an element is zero. This condition is aptly called the Incidence Law. Generally, it is stated in the simpler form; "the algebraic sum of all through variables leaving a junction point is zero." The foregoing statements show that a through variable is an oriented quantity; it can be transmitted in either of two directions.

Across variables obey a different law. Such a variable is basically, and by definition, the difference of two scalar quantities, each scalar quantity being associated with a specific terminal. The terminal associated with the scalar quantity which is subtracted is called the Reference Terminal for that variable. Thus every across variable is an oriented quantity in the sense that its definition must specify which of two terminals is the reference. If the algebraic sum of across variables associated with a mesh is computed in terms of the scalar quantities at terminals it is easy to show that the result is always zero. Thus across variables satisfy the Mesh Law; namely that the algebraic sum of across variables around a mesh is zero. It is well to note that whereas the Mesh Law can be proven from first principles, the Incidence Law is only a statement of experience.

At this point an analyst has in hand a set of elements each with identified terminals, an independent set of across variables, a coded graphical symbol for the element, and a set of constitutive equations. If he has done his work systematically, each element terminal corresponds to one, and only one, terminal in the original connected system. It is to be noted that whereas each element terminal corresponds to only one system terminal, which hereafter shall be called a Junction Point, each junction point can, and usually does, correspond to more than one element terminal.
In other words, a junction point is a coincidence of several element terminals. Using these facts the analyst can generate easily a schematic diagram for the connected system by placing circles, say, on a sheet of paper; each circle corresponding to one specific junction point in the system. Graphical symbols for the elements are now connected between the junction points so that each element terminal is made coincident with the junction point to which it corresponds. This process amounts to showing graphically how the across variables satisfy the mesh laws. A more engineering like statement is that the process specifies the connectivity existing among the elements.

The foregoing process may lead to a schematic diagram with a congested, or messy, appearance. Engineers have techniques for improving the appearance of these diagrams; but of these only one is of concern to us today. This technique rests upon the fact that if it is known that the across variable defined between two supposedly different junction points is always zero, then in fact the two junction points are one and the same. Such a situation is shown in a schematic diagram by an uncoded line segment connected between the two supposedly different junction points. If desired the analyst could show that the two separate junction points are in fact the same by shrinking the uncoded line segment to zero. The process can be reversed. If a junction point has many line segments incident upon it, thus leading to a congested diagram, this single junction point can be represented in the schematic diagram as several junction points joined by uncoded line segments. Of course, this separation must be done so that all mesh relations are still satisfied and no new meshes are introduced into the diagram.

At this point the following can be asserted, "To every finite engineering system made up of elements whose constitutive equations are known, there exists a schematic diagram which specifies the connectivity of the systems and the constitutive equations for each element." The truth of this statement is obvious since a process for creating such a diagram has been defined. The principle is worded to show that if a system cannot be conceptually decomposed into elements whose terminals and constitutive equations are known then such systems can not be diagramed or analyzed at the moment. This situation exists for example with systems in which thermal energy is converted to some other form, and vice versa.

It is important to note that the mesh and the incidence laws, which are often termed the Feld Equations, are linear in the algebraic sense. Constitutive equations on the other hand need not be linear and the exciting situations for the future are the nonlinear ones. This difference cannot be emphasized too much for it is a rare person indeed who keeps these two items separated in his mind and in his analyses. Note that the field equations concern themselves only with the connectivity of the system; they have nothing whatsoever to do with the constitutive relations.

If one can wax philosophical for a moment, it may be pointed out that there are only two ingredients in- 
volved in establishing descriptive equations for a system; these are the connectivity of the system and the constitutive equations of the elements. These two types of information correspond to the two engineering operations of selecting some devices from a bin, shelf, or ordering them from a supplier and then riveting, soldering, gluing, or otherwise joining them together. That is all there is to creating an engineering system. Thus it is seen that the generation of correct descriptive equations for a system can involve at most the connectivity of the system and the constitutive equations. Our task is to show how connectivity can be specified and how it can be made to react on the constitutive equations to yield equations for the system as a whole.

An analysis of an engineering system involves two distinct and separate phases; the formulation and the solution phase. All of the physical and engineering principles needed to deal with the system are used in the formulation phase. A bit of mathematics is used in this phase also. The more difficult mathematics is involved in the second phase, that is in solving the equations already formulated. This phase is also the most time consuming. It is a fact that generally speaking, we have mathematical tools for solving linear equations, but not for nonlinear equations. But if system equations are linear, so also are the constitutive equations for the elements. In such a situation we have linear field equations, linear constitutive equations, and linear system equations. It is not surprising therefore that these items have become confused in the minds of workers. This is not a logical position to assume and furthermore it severely restricts one's ability to deal with these exotic nonlinear engineering systems which are appearing in ever increasing numbers these latter years.

Let us now make a closer contact with the theory of linear graphs. Let a schematic diagram be given for an engineering system. In this diagram let each uncoded line segment be shrunk to zero. Finally let the coding be removed from each line segment. The result is a geometrical graph in which each vertex has a l-to-1 correspondence to a conceptual junction point in the system and each edge, or arc, has a l-to-1 correspondence to one across and one through variable. We now seek properties of the graph which follow the same algebraic laws as the across and through variables, i.e., the mesh and incidence laws. Again it is emphasized that these are linear laws. Let it be stated for emphasis that the linear graph has a correspondence only with the field equation of a system, not to its constitutive relations. These latter relations correspond to the coding of a schematic diagram; but all coding has been removed. It is not difficult to identify those properties of a graph that are needed. Since the theory is rather elementary and known to nearly everybody in this audience, only the highlights of the argument will be given.

Every edge in a graph has a boundary, say $\partial e_{\mathrm{i}}$. Let the collection of all edge boundaries be represented by the row vector $\partial \mathrm{E}$. It is well known that the elements of $\partial E$ belong to a linear vector space. Hence any object in the space is given by a relation of the form $e_{i}^{*}=\partial E \eta$ where $\eta$ is a column vector whose elements are taken from the field of rational numbers. Furthermore an independent set of new objects can be defined by a matrix relation of the form $\partial E^{*}=\partial E K$ where $K$ is a nonsingular matrix with rational numbers as elements.

Each edge also provides an incidence, on two vertices. Let this be $\delta e_{i}$ if the edge $e_{i}$ is oriented away from the vertex and $-\delta e_{i}$ if $e_{i}$ is oriented toward the vertex. The collection of all edge incidences will be represented by the column vector $\delta \bar{E}$.

Each vertex can be viewed either as providing a part of the boundaries of some edges or as having an incidence because of them. The first notion for a vertex will be represented by $\hat{v}_{j}$ and the second by $\bar{v}_{j}$. Let the boundary property of vertices be collected together into the row vector $\hat{V}$ and the incidence properties into the column vector $\bar{V}$.

Since the time of Poincare it has been known that $\partial E=\hat{V} \pi$ and $\bar{V}=\pi \delta \mathrm{E}$ where $\pi$ is a rectangular matrix. The elements of $\partial E$ are said to correspond to the elements of $\delta \mathbf{E}$. Suppose that the first relation is post multiplied by $K$ giving $\partial E K=\partial E^{*}=\hat{\nabla} \pi K=\hat{V} \pi^{*}$. The corresponding elements from a new set of incidence objects can be found by using the principle that the incidence on any vertex is independent of the set of incidence objects used as a basis. Thus $\bar{V}=\pi \delta E$ $=\pi\left(K K^{-1}\right) \delta E=(\pi K)\left(K^{-1} \delta E\right)=\pi^{*} \delta E^{*}$ where $\delta E^{*}$

$=K^{-1} \delta E$. These facts give rise to the invariance principle $\partial E \delta E=\partial E^{*} \delta E^{*}$.

A mesh is defined to be a graphical object that has no boundary. Thus a mesh is specified by a column vector $\eta$ which satisfies $\partial E \eta=\hat{V} \pi \eta=0$. Thus $\pi \eta=0$ for a mesh. There are an unlimited number of solutions to this equation but it is easy to show that the number of independent solutions is $\epsilon-\nu+\mathscr{f}$ where $\epsilon$ is the number of edges in the graph, $\nu$ the number of vertices and $\mathcal{S}$ the number of disjoint parts. Call this number $\mu$, the number of independent meshes.

Hereafter $K$ will be used only in the so-called canonical form in which the first $\mu$ columns define $\mu$ independent meshes. Thus $K$ can be written in the partitioned form $K=|M| P \mid$ where $M$, the so-called mesh connection matrix is the first $\mu$ columns. Since $K$ is nonsingular it has an inverse which can be written in the conformally partitioned form $K^{-1}=\left|\frac{Q^{\prime}}{N^{\prime}}\right|$ where $N$ is the so-called nodal connection matrix and the primes denote transposition. If $\partial E^{*}$ and $\delta E^{*}$ are partitioned conformally according to the scheme $\partial E^{*}=\left|\partial E_{1}^{*}\right| \partial E_{2}^{*} \mid$ and $\delta E^{*}=\left|\frac{\delta \mathrm{E}_{1}^{*}}{\partial E_{2}^{*}}\right|$ it is easy to show that $\partial E=\partial E_{2}^{*} N$ and $\partial E_{1}^{*}=0$ since the objects which give rise to the elements of $\partial E_{1}^{*}$ are all meshes.

It is clear that the boundaries of edges satisfy the mesh law. Hence using the fact that there is an across variable which corresponds to each edge it is clear that each across variable corresponds algebraically to the boundary of its corresponding edge and that $\partial E$ can be replaced by $A^{\prime}$ where $\mathrm{A}$ is a column vector of across variables. For example $\mathrm{A}=N A_{2}^{*}$.

In a similar fashion, $\delta E$ can be replaced by a column vector $T$ where the elements of $T$ are through variables, 
but at the same time, it is necessary to impose the incidence laws. That is, to each element in $\bar{V}$ we set in correspondence a scalar, say $i_{k}$, and let the collection of elements be written $I$. Thus we have $I=\pi T=\pi K T^{*}$

$=\pi|M| P|| \frac{T_{1}^{*}}{T_{2}^{*}} \mid=\pi M T_{1}^{*}+\pi P T_{2}^{*}=\pi P T_{2}^{*}$ since $N M=0$

by definition. Now the elements of $I$ are set equal to zero to correspond to the incidence laws. It can be shown that $\pi P$ is nonsingular and hence that $T_{2}^{*}=0$. Since $T=K T_{2}^{*}=|M| P|| \frac{T_{1}^{*}}{T_{2}^{*}} \mid$ it follows that $T=M T_{1}^{*}$.

Using the results to date, it is important to observe the invariance principle yields $A^{\prime} T=A_{2}^{* \prime} N^{\prime} M T_{1}^{*}=0$ since $N^{\prime} M=0$ as is easily shown from the relation $K^{-1} K=I$. This principle says that for any engineering system the formal matrix product of the across and through variables for the system is always zero. One specialized interpretation of this principle is that all of the energy supplied to a system is either stored or dissipated, a rather obvious result. It is strange that the principle is not well known since it is so basic; in fact it would appear that few people indeed have even heard of it. It is even stranger that it was only a few years ago that the principle was proved in the special case of electric networks by an engineer, at the Phillips company in Holland. Only by the argument given here has it been proved for all other cases.

Let us now consider the constitutive equations for a system. It is usually possible to write this in a matrix form like $\Lambda A=T+T_{g}$ where $\Lambda$ is an operator matrix and $T_{g}$ is a column vector of through variables delivered by the sources in the system. Now let the equation $A=N A_{2}^{*}$ be used to eliminate $A$ giving $\Lambda N A_{2}^{*}=T+T_{g}$. Finally let the relation be premultiplied by $N^{\prime}$ giving $N^{\prime} \Lambda N A_{2}^{*}=N^{\prime} T+N^{\prime} T_{g}=N^{\prime} T_{g}$ since $N^{\prime} T=N^{\prime} M T_{1}^{*}=0$. This equation gives the so-called nodal formulation. It should be observed that if $\Lambda$ contains some nonlinear operators care must be taken in handling the indicated operations in order to get correct results.

A parallel type of development yields the so-called mesh formulation. Let the constitutive equations be written in the form $\psi T=A+A_{g}$ where $\psi$ is a matrix of operators and $A_{g}$ is a column vector of across variables supplied by sources. Let $T=M T_{1}^{*}$ be substituted and let the result be premultiplied by $M^{\prime}$ giving $M^{\prime} \psi M T_{1}^{*}$ $=M^{\prime} A+M^{\prime} A_{g}=M^{\prime} A_{g}$ since $M^{\prime} A=M^{\prime} N A_{2}^{*}=0$ since $M^{\prime} N=0$.

In either case the constitutive relations enter through an operator, $\Lambda$ or $\psi$, and a vector of variables specified by sources, either $T_{g}$ or $A_{g}$. The connectivity of the system is specified by $N$ in one case and $M$ in the other; the descriptions are equivalent. Thus it is seen that the connectivity of the system reacts on the constitutive equations by a congruence transformation to give a correct set of equations for the system as a whole.

If the engineering system under analysis involves only one energy mechanism which is to be analyzed in detail the foregoing procedures are adequate to handle the formulation phase. We shall let the professional mathematicians worry about the second or solution phase.
If, however, the system involves two or more energy mechanisms, each of which is to be analyzed in detail, some extra complications arise. Time will not allow a detailed discussion of this case. Since a substantial fraction of modern and interesting systems are of this type it is a bit of a pity that we cannot go into such analyses in detail. The subject is important and is worthy of a lengthy discussion on its own merits. We shall only say here that the concept of a perfect coupler, with its two types, is involved here; where by a perfect coupler is meant a conceptual mechanism which can neither create, store, nor dissipate energy but can transfer it from one part of the system to another, often changing its type in the process. These conceptual elements perform their function by imposing constraints on the variables. The imposition of these constraints on the equations obtained by the previous technique is not a hard mathematical problem, but will not be covered today. Suffice it to say that these extensions allow us to treat any system, no matter how complicated, if we know the connectivity of the system and the constitutive equations of the elements.

If most of you feel that the material I have presented today is almost insultingly simple then my lecture has been a success. I can only hope that this is the case for after all many of the important sets of concepts and procedures in the natural sciences are basically simple. Once a proper set of concepts has been assembled and extraneous matters eliminated we are left with a simple but powerful technique for making the formulation of system equations almost a routine process.

\section{Some Related Publications by Dr. Trent:}

1. Generalized automorphisms of labelled graphs and graphic invariants, with L. Auslander, J. Math Phys. XLII, No. 1 (Mar. 1963).

2. A decomposition theorem concerning linear graphs, J. Math Mech. 13, No. 2 (Mar. 1964)

3 . On the realization of a linear graph given its algebraic specification, with L. Auslander, J. Acoust. Soc. Am. 33 (Sept. 1961).

4. On the topology of printed circuits, with L. Auslander, IRE Transcations CT-6 (Dec. 1959).

5. Incidence matrices and linear graphs, with L. Auslander, J. Math. Mech. 8 (Sept. 1959).

6. On the conceptual necessity and use of perfect couplers in schematic diagrams, J. Acoust. Soc. Am. 31 (Mar. 1959).

7. On the construction of schematic diagrams for mechanical systems, J. Acoust. Soc. Am. 30 (Aug. 1958).

8. Isomorphisms between oriented linear graphs and lumped physical systems, J. Acoust. Soc. Am. 27 (May 1955).

9. A note on the enumeration and listing of all possible trees in a connected linear graph, Proc. Natl. Acad. Sci. 40 (Oct. 1954).

10. An equivalent circuit for a vibrating beam which includes shear motions, J. Acoust. Soc. Am. 22 (May 1950).

11. The Laplace transform as a form of curve fitting, Am J. Phys. 17 (1949).

12. The absolute calibration of electromechanical pickups, J. Appl. Mech. (Mar. 1948).

13. Reciprocality in transducers, J. Acoust. Soc. Am. 19 (May 1947).

14. Ultrasonic measurement of wall thickness in diesel cylinder liners, J. Acoust. Soc. Am. 19 (Mar. 1947).

15. The detection of internal leaks in aircraft hydraulic systems, with R. G. Nuckolls, J. Acoust. Soc. Am. 19 (Mar 1947). 
16. Some discrepancies in electromagnetic theory, Proc. Miss. Acad. Sci. (1940).

17. The concept of pressure energy, Proc. Miss. Acad. Sci. (1939).

18. The fourth dimension in electrical theory, Proc. Miss. Acad. Sci. (1939).
19. Diaphragmless microphones, Proc. Indiana Acad. Sci. (1935). 20. A study of the velocity of sound in solutions, Proc. Indiana Acad. Sci. (1935).

(Paper 69B1-136) 